\title{
A communication model with limited information-processing capacity of recipients
}

\author{
Oleg V. Pavlov, ${ }^{a}$ Robert K. Plice ${ }^{\mathrm{b}}$ and Nigel P. Melville ${ }^{\mathrm{c}}$
}

\begin{abstract}
Oleg Pavlov is Associate Professor of Economics and System Dynamics at Worcester Polytechnic Institute (WPI), U.S.A. Professor Pavlov has published on such topics as electronic marketplace (peer-topeer networks and electronic marketing), macroeconomic growth, governance, telecom and stock market dynamics. He is past President of the Economics Chapter of the International System Dynamics Society. He earned a BS in Physics and Computer Science and a $\mathrm{PhD}$ in Economics from the University of Southern California. Before joining WPI, he was a postdoctoral fellow in Information Systems at Boston University School of Management.
\end{abstract}

Robert Plice is Associate Professor of Information and Decision Systems at the College of Business Administration, San Diego State University. Professor Plice researches information technology and its impact on organizations and

\begin{abstract}
We develop a system dynamics model of message-based communication, where the informationprocessing capacity of message recipients is limited. Profit-seeking broadcasters send messages, but only some of these messages are valuable to recipients. Recipients cannot determine whether or not a message is valuable until it is processed. Information overload occurs when more messages arrive than recipients can process. Numerical experiments test alternative approaches for mitigating information overload. We show that message filtering can increase the flow of for-profit communication. Market-based mechanisms, while aimed at improving the social outcome, can actually lead to suboptimal results and to a complete collapse of for-profit communication. Copyright @ 2008 John Wiley \& Sons, Ltd.
\end{abstract}

Syst. Dyn. Rev. 24, 377-405, (2008)

\section{Introduction}

Electronic mail has become an important component of the digital marketing portfolio for many organizations (Gatti, 2004a). A motivation for the wide adoption of direct online marketing is the superior return on investment, as compared to any other type of marketing. According to the Direct Marketing Association, the return on investment for commercial e-mail is about $\$ 50$ for every dollar spent, while catalog sales are only about $\$ 7$ for every dollar spent (Magill, 2007). The exceptional return is possible due to the high degree of customization (Rust and Espinoza, 2006) and very low cost of e-mail communication.

From a social standpoint, a major drawback of commercial e-mail, also called spam, is that it imposes both direct and indirect costs on the receiving side. The direct cost entails the infrastructure needed to support a high flow of commercial e-mail and filters that sort through the incoming messages. The indirect cost results from the attention that the recipients must dedicate to processing commercial messages. Inbox clutter due to spam has led to a strong sentiment in society against commercial e-mail. Some even go so far as to say that "spam has ruined the Internet" (Fallows, 2003).

\footnotetext{
a Department of Social Science and Policy Studies, Worcester Polytechnic Institute, 100 Institute Road, Worcester, MA 01609, U.S.A.

${ }^{\mathrm{b}}$ Information and Decision Systems, San Diego State University, San Diego, CA, 92182, U.S.A.

c Stephen M. Ross School of Business, University of Michigan, Ann Arbor, MI, 48109, U.S.A.

* Correspondence to: Oleg V. Pavlov. E-mail: opavlov@wpi.edu

Received December 2007; Accepted June 2008
}

System Dynamics Review Vol. 24, No. 3, (Fall 2008): 377-405

Published online 13 November 2008 in Wiley InterScience

(www.interscience.wiley.com) DOI: 10.1002/sdr.407

Copyright (c) 2008 John Wiley \& Sons, Ltd. 
workforces. He is the author of research papers that have appeared in academic and professional journals such as Communications of the ACM, Communications of the AIS, and Journal of Organizational Computing and Electronic Commerce. He earned a BA in Economics from American University, an MS in Computer Science from the University of Maryland, a BS in Business Administration from San Diego State University, and a PhD in Management from UC Irvine.

Nigel Melville is an Assistant Professor of Business Information Technology at the Stephen M. Ross School of Business, University of Michigan. Professor Melville is an expert in information technology innovation and organizational performance. He is the author of numerous research articles appearing in leading academic and professional journals, such as Information Systems Research, MIS Quarterly, Decision Support Systems, and Communications of the ACM. He is an
Despite the efforts of regulators, Internet service providers (ISPs) and the vendors of anti-spam products, global spam production has been increasing. There are many players in the spam industry-including software companies, e-mail harvesters, spammers, sellers and ISPs (Scalet, 2003; McWilliams, 2005; Claburn, 2008)—who have vested interests in continuance of e-mail spam. In 2007, marketers spent about $\$ 500$ million on e-mail advertising and generated about $\$ 23$ billion in sales (Magill, 2007). There is also a thriving spam-fighting industry: a $\$ 140$ billion a year business (Kleiner, 2008).

The problem of unwanted commercial messages is significant on other communication platforms (Marshal, 2007), such as instant messaging (spim), blogs, forums and mobile text messaging. Spammers are known to use bogus accounts on social websites, such as Facebook and MySpace (Kleiner, 2008). Even cellular telephones are affected by unsolicited commercial messages, exemplified by Verizon's filing of a lawsuit in 2006 against a spam company.

In this paper, we model the mechanism common to spam, spim and any other type of commercial communication that depends on the limited information-processing capacity of recipients. A generic model of communication is used to test proposed remedies for decreasing the flow of marketing messages. We show that reliance on filtering will not, by itself, solve the spam problem. Market-oriented solutions offer a much better alternative, but may lead to market failure. The findings add to the current public policy debate about the direct online marketing industry. E-mail, the inspiration for this paper, is a common good (Regan, 2002; Pavlov et al., 2005), which makes the analysis of this paper also relevant to the ongoing discussions about digital commons (Stix, 2002; Hunter, 2003; Greco and Floridi, 2004) and their management (McFadden, 2001).

The next section gives some facts about direct marketing, including spam. The section also reviews mitigation methods. The discussion suggests several reference modes. In the following two sections, we outline our dynamic hypothesis and present a system dynamics model. In the Policies section, we investigate the effects of the proposed mitigation mechanisms. We conclude with a discussion of our research results.

\section{Reference modes}

\section{Possible scenarios}

Spam industry observers agree that spam production has been growing over the years (The Economist, 2004; Kleiner, 2008). It was under 10 percent of the global e-mail traffic in 2000, but now spam constitutes over 90 percent of global e-mail traffic. Therefore, the feared scenario is that overall spam production may increase (Figure 1). 
Fig. 1. Reference mode for possible scenarios editor of the book Global E-Commerce: Impacts of National Environment and Policy (Cambridge University Press, 2006). Professor Melville is also a Special Sworn Status researcher of the U.S. Census Bureau at the Michigan Research Data Center, University of Michigan. Previously, Professor Melville was the Sam M. Walton SIFE Free Enterprise Fellow and Assistant Professor of Information Systems at the Wallace E. Carroll School of Management, Boston College. Professor Melville earned a BS in Electrical Engineering from UCLA, an MS in Electrical and Computer Engineering from UC Santa Barbara, and a PhD in Management from UC Irvine.

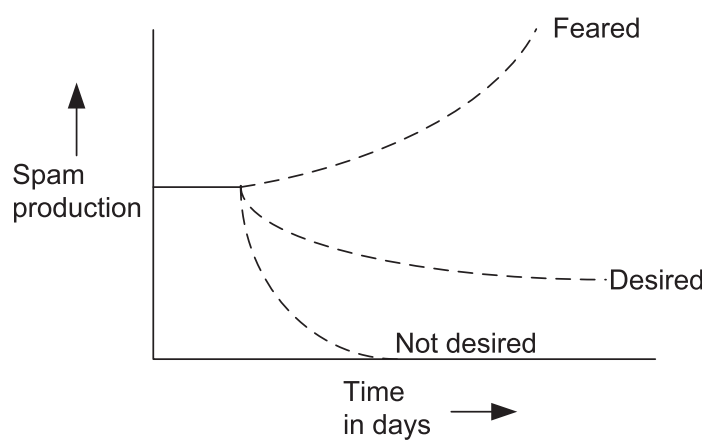

While a reduction in spam production might be desirable, an all-out elimination of spam (if it were possible) would not be socially optimal (e.g., Sweet, 2003; Dai and Li, 2004; Goldman, 2006). First, to maximize social welfare, some spam should be allowed (Loder et al., 2006). Second, spam also provides an inexpensive communication tool for political and grass-roots organizations (Sweet, 2003). A desired scenario would be a reduction in spam production, but not its complete elimination (Figure 1).

\section{Response rate}

Traditional marketers are aware of the negative relationship between the advertising volume sent to a group of recipients and the response rate (Rudolph, 1947; Starch, 1966; Houston and Scott, 1984). Recent research suggests that the same negative relationship holds for electronic markets. For instance, Martin et al. (2003) found that in the case of a permission-based e-mail campaign, the likelihood of visiting a link advertised within an e-mail decreased as volume of e-mail from the company increased. Similarly, a survey by the Pew Internet \& American Life Project (Rainie and Fallows, 2004) found a decline in the readership of marketing e-mails as their volume increased. Anecdotal evidence from spammers (Hansell, 2003) also confirms the existence of a negative relationship between the amount of spam a group receives and its response rate. A system dynamics model that simulates marketing response should reproduce the negative pattern (Figure 2(a)).

\section{Filtering}

The most widely deployed tool for controlling unwanted spam is filtering. A survey found that 62 percent of online users rely on spam filters (Gatti, 2004c). The function of a spam filter is to identify and divert spam before it reaches the addressee's inbox. Filtering can be very effective at blocking all kinds of commercial messages, as acknowledged by spammers themselves (McWilliams, 2005 , p. 89). The benefit of filtering is that it reduces demand for the attention 


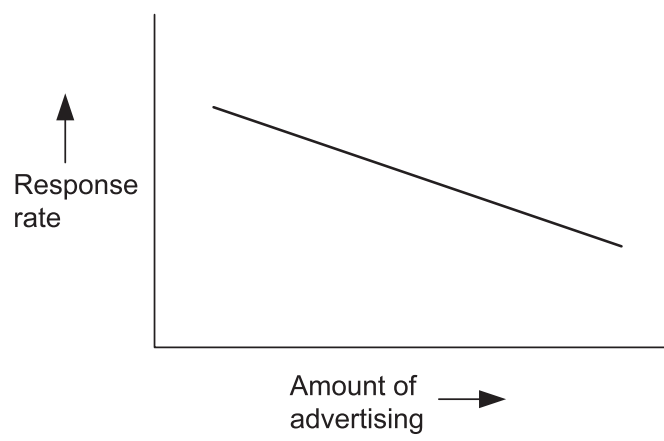

(a)

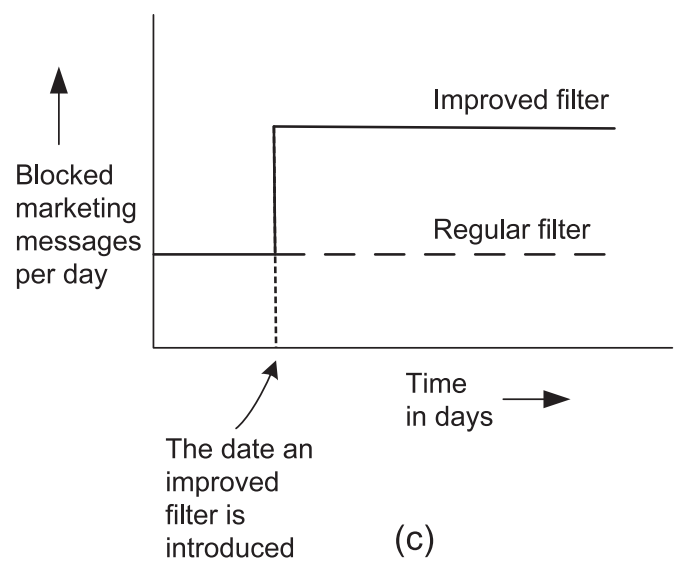

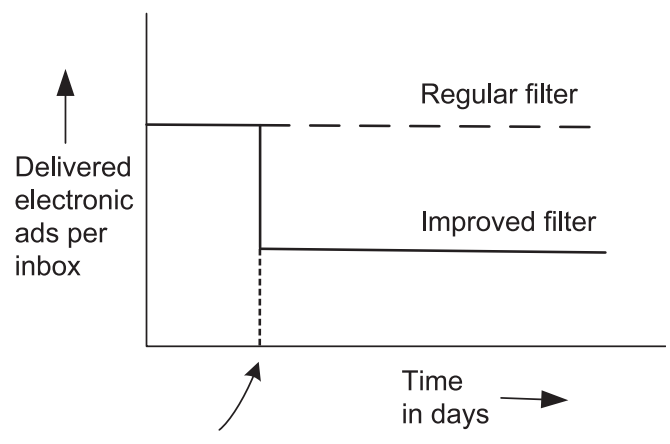

The date an improved filter is introduced

(b)

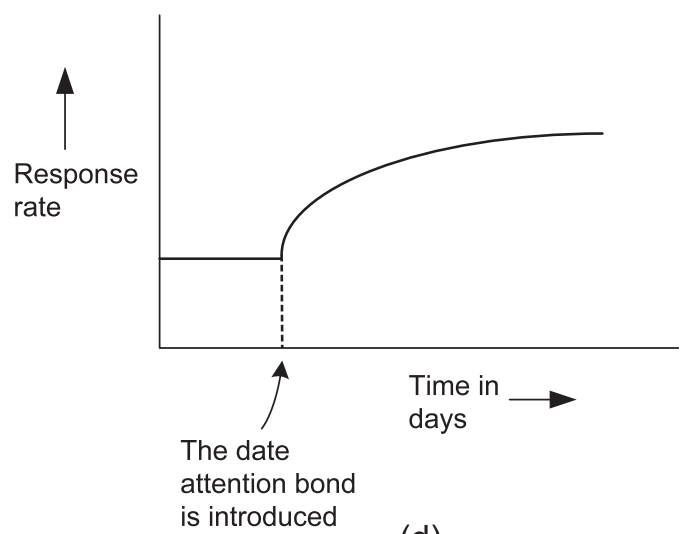

(d)

Fig. 2. Reference modes: (a) response rate and ad exposure; (b) effect of an improved filter on spam delivery; (c) effect of an improved filter on message blocking; (d) claimed effect of attention bond on response rate

of the e-mail user. The filtering solution seems to be working, to the extent that users report a lesser burden of spam at work than on their personal e-mail accounts because of active e-mail screening at work (Fallows, 2003). This fact suggests that a flow of commercial messages to individual inboxes depends on the filtering effort (Figure 2(b)).

A downside of the technology is that filters are costly (in terms of time and money). Plus, the cost falls on the recipients, rather than the senders, of spam. Another drawback is that filters are imperfect: the software can mistakenly identify legitimate e-mail as spam (false positive) and let spam get through to the inbox (false negative). For example, in one study, the SpamCatcher software classified 1.1 percent of legitimate e-mail as spam, i.e. false positives, and allowed 12 percent of spam into inboxes, i.e. false-negatives (Rubenking, 2004). Marketers report that between 10 and 17 percent of permission-based 
commercial e-mail is blocked (Gatti, 2004a, 2004b). A survey found that among those who use filters, only 17 percent think filters work very well, 25 percent reported that filters block wanted messages, and 48 percent reported that filters let through excessively many unwanted messages (Gatti, 2004c). An improved filter should be able to block more unwanted marketing messages (Figure 2(c)).

\section{Market mechanisms}

Because of the many problems with filtering, there is an active interest in exploring market-based solution. Market-based solutions aim to mitigate the misallocation of the full cost associated with spam by aligning the private cost more closely with the social cost. There are two major proposals: the electronic postage surcharge and an attention bond mechanism.

The electronic postage scheme (Kraut et al., 2002; Van Zandt, 2004; Loder et al., 2006) envisions charging commercial marketers a fixed surcharge for each message they sent. Microsoft's Bill Gates has been known to support e-mail postage (The Economist, 2004). One of the first working implementations of such a surcharge system is offered by a company named Goodmail (http://www.goodmailsystems.com/; last accessed 23 May 2008). In the Goodmail system, a client company purchases electronic security "strips" in bulk from Goodmail. Proprietary software attaches the "strips" to the outgoing e-mails of the client. Goodmail has enlisted several ISPs that do not block an e-mail if the e-mail contains the security "strip" attached to the heading of the message. Companies such as Petco and Godiva Chocolatier are among Goodmail's customers.

Fahlman (2002) advanced a concept of interrupt rights for e-mail or phone calls, which Loder et al. (2006) call an attention bond mechanism. Its economic logic is to offer monetary compensation to the person who bears the cost of an interruption from an incoming communication (The Economist, 2004). The schema resembles the direct marketing practice of inducing customer response by offering a financial incentive with a marketing message (Turley, 1999; Chittenden and Rettie, 2003; EmailLabs, 2006). One of the earliest attention bond mechanisms was implemented and offered by Return Path, under the name Bonded Sender Program. Return Path claimed that, on average, response rates for marketers who participated in the program improved significantly (Return Path, 2006). Figure 2(d) shows the expected improvement in response rates under the attention bond scheme as a reference mode.

\section{Dynamic hypothesis}

The commercial e-mail industry is driven by the promise of profit (Scalet, 2003; McWilliams, 2005; Claburn, 2008). Profit, also called surplus, determines the stock of communication funding available for marketing campaigns (Figure 3). 


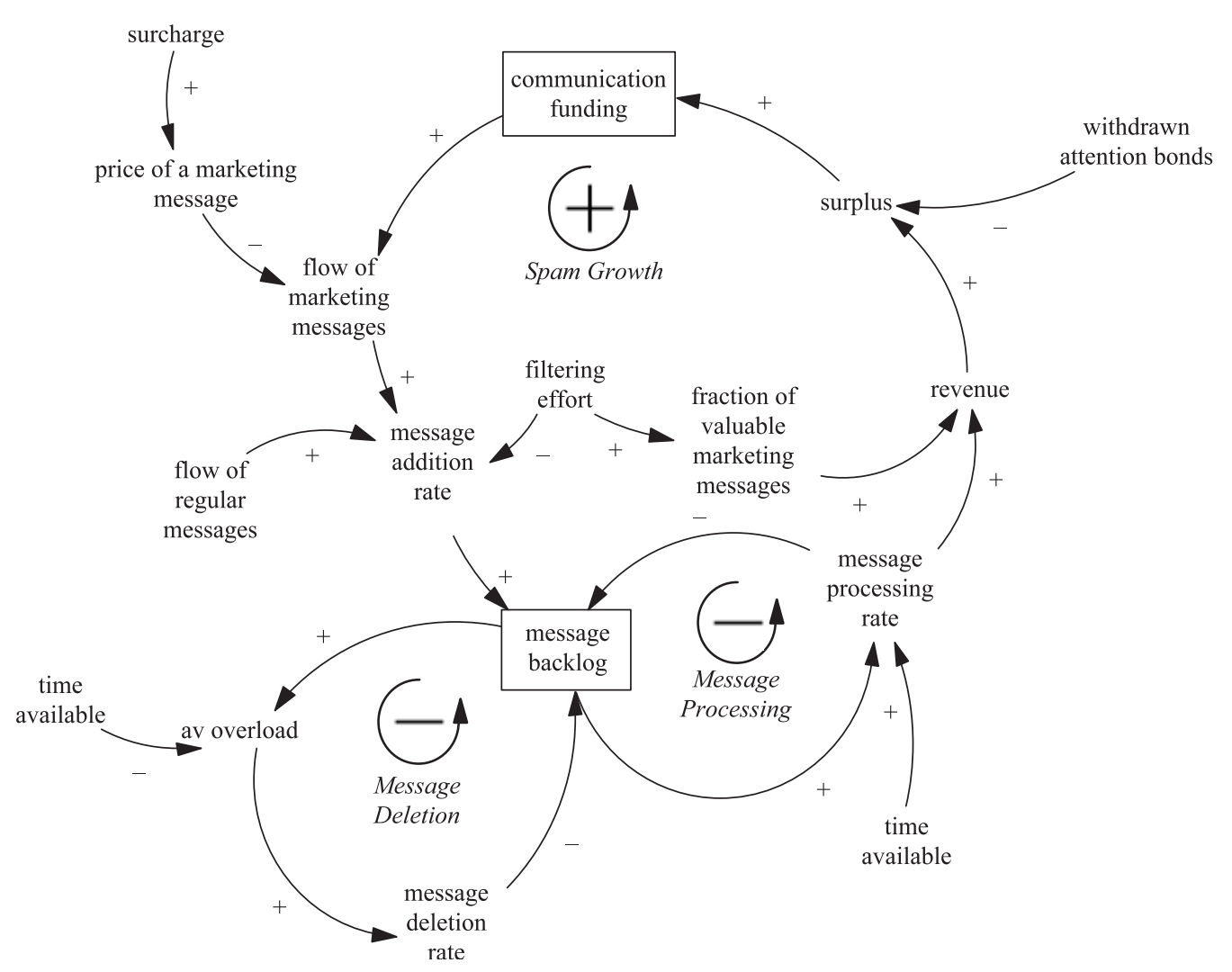

Fig. 3. A dynamic hypothesis

More money for communication increases the flow of marketing messages. The higher the price of sending a marketing message, the lower the marketing messages flow is. The flows of regular and marketing messages drive the message addition rate to the message backlog.

The messages in the backlog can be either processed or deleted. The message processing rate depends on the backlog and the time available for processing. Processing messages relieves the backlog, and hence a negative link between the message processing rate and the stock message backlog. But as the message backlog grows, so does the information overload the recipients experience. As the recipients feel overburdened with messages, they delete an increasing number of messages before opening them. Deleting messages reduces the message backlog.

As messages from the backlog are processed, the recipients might find some of the commercial messages valuable. Recipients respond to valuable marketing messages, thus generating revenue for spammers. The greater the fraction of valuable marketing messages in the message backlog, the more valuable marketing messages are processed and more revenue is generated. The positive 
relationship between revenue, message processing rate and fraction of valuable marketing messages is shown as positive links in Figure 3.

The causal links form three loops: the reinforcing Spam Growth loop, the balancing Message Processing loop and the balancing Message Deletion loop. The Spam Growth loop drives spam production, checked by the balancing loops. A minor balancing loop, Message Processing, controls the message backlog, while recipients have not exhausted their time available. When recipients see that the message backlog is greater than they can process, the Message Processing loop can no longer stop message backlog growth. At that point, the balancing Message Deletion loop must slow down the growth of the backlog.

An examination of the diagram in Figure 3 reveals that the causal structure is an extension of a well-known limits-to-growth generic structure (Senge, 1990). The limits-to-growth structure characteristically has at least one reinforcing loop and at least one balancing loop dependent on some limiting constraint. In our case, attention-modeled as time available-is the limiting constraint. As is typical of limits-to-growth structures, we expect the structure in Figure 3 to generate S-shaped trajectories for our stock variables.

The filtering effort has two effects (Figure 3): it blocks some messages, and therefore reduces the message addition rate. By blocking unwanted messages, the filter also improves the fraction of valuable marketing messages in the backlog. If an electronic postage surcharge is introduced, it will directly add to the price of sending a marketing message-just as higher regular postage adds to the price of mailing a letter. When recipients withdraw attention bonds, the surplus of spammers is reduced.

\section{Model}

The spam industry is populated by highly specialized economic agents, each performing a distinct role (Scalet, 2003; McWilliams, 2005; Claburn, 2008). This model, however, simplifies reality by considering only five types of players, all conveniently aggregated and organized into model sectors (Figure 4). The sectors are: Recipients, Sponsors, Broadcasters A, Broadcasters B and the Information Technology (IT) Company.

Because the topic of this paper is relevant to many information systems (IS) platforms (e-mail, text messaging, blogs, etc.), we model communication in generic terms. We refer to messages as type-A and type-B. Type-A messages are regular messages, valued by their Recipients. Type-B messages are marketing messages; only a fraction of them is valuable to Recipients. Within the e-mail context, type-A messages are regular e-mail messages and type-B messages are spam. The messages are produced correspondingly by Broadcasters A and Broadcasters B. When Recipients respond to marketing messages, Sponsors receive revenue. Sponsors then pay Broadcasters B to send more type-B messages. The IT Company provides Recipients with a filter. 
Fig. 4. Subsystem diagram

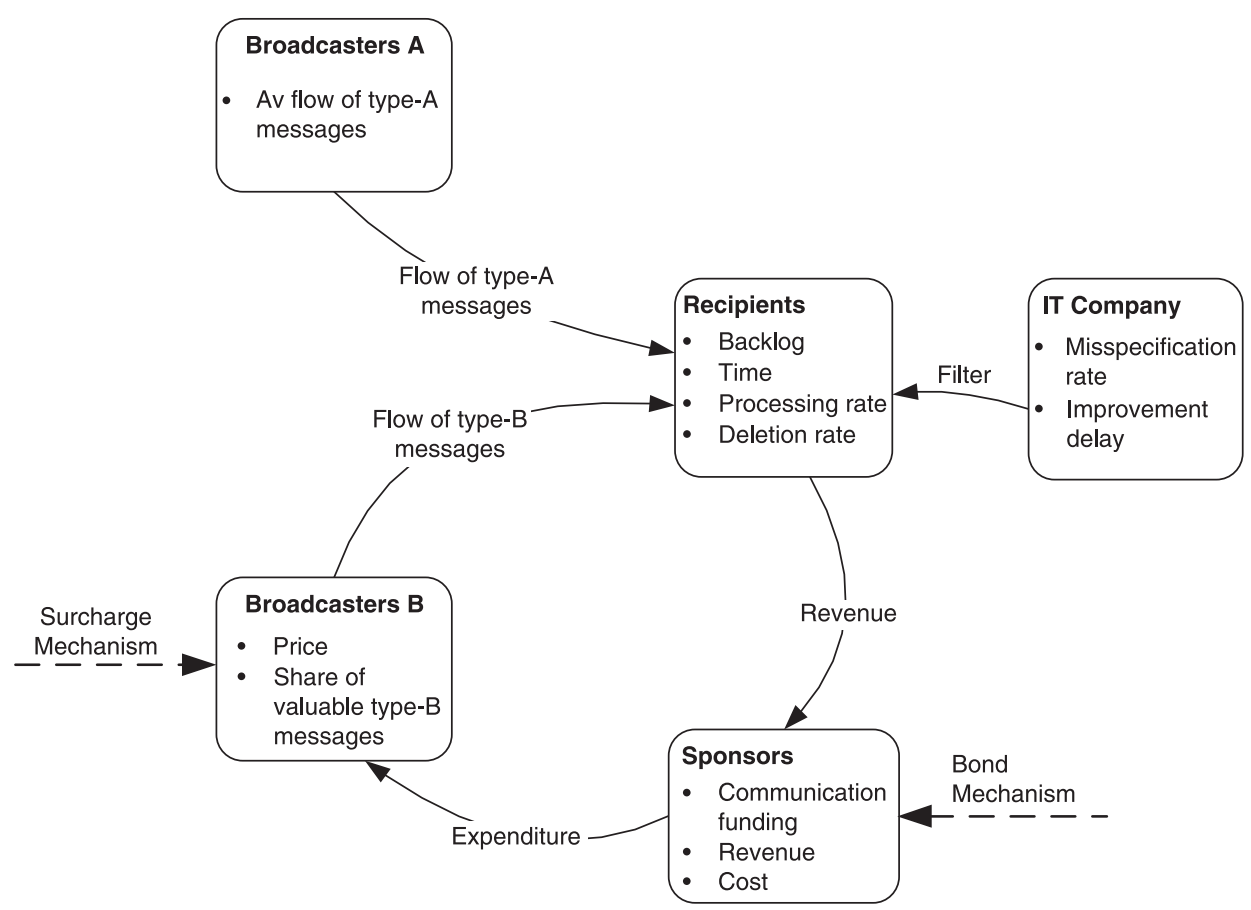

This paper is concerned with the global trend in marketing communication. Therefore, rather than modeling individual inboxes, we aggregate them as a message backlog in the Recipients sector. We can think of the Recipients sector as a representation of, for example, either a national market or an ISP with many customers. A defining feature of such a group of customers is that their collective attention, regardless of how large the group is, is a scarce economic resource (Davenport and Beck, 2001).

One can think of several ways to implement marketing mitigation mechanisms. We assume that the burden of surcharges, if introduced, falls directly on Broadcasters B (the spammers) and, if the bond mechanism is instituted, then Sponsors have to provide the bond payments.

Unlike previous research on the spam problem (e.g., Van Zandt, 2004; Khong, 2004; Anderson and De Palma, 2005; Loder et al., 2006), we construct a system dynamics model. The model has been implemented in Vensim DSS. We set the time horizon as one year (365 days). In the following sections we describe the model sector by sector.

\section{Recipients}

Within the Recipients sector, two types of messages-type-A messages (A messages, for short) and type-B messages (B messages)-are added to the 
Fig. 5. Recipients receive two types of messages. Messages from the message backlog can be either processed or deleted aggregate message backlog (Figure 5). As a group, Recipients can either process messages from the backlog or delete them. This subsection describes in detail the system dynamics formulation of the Recipients sector.

Message backlog

We do not differentiate between individual recipients but, rather, we consider an aggregate backlog $M$, comprised of messages in the inboxes of all recipients.

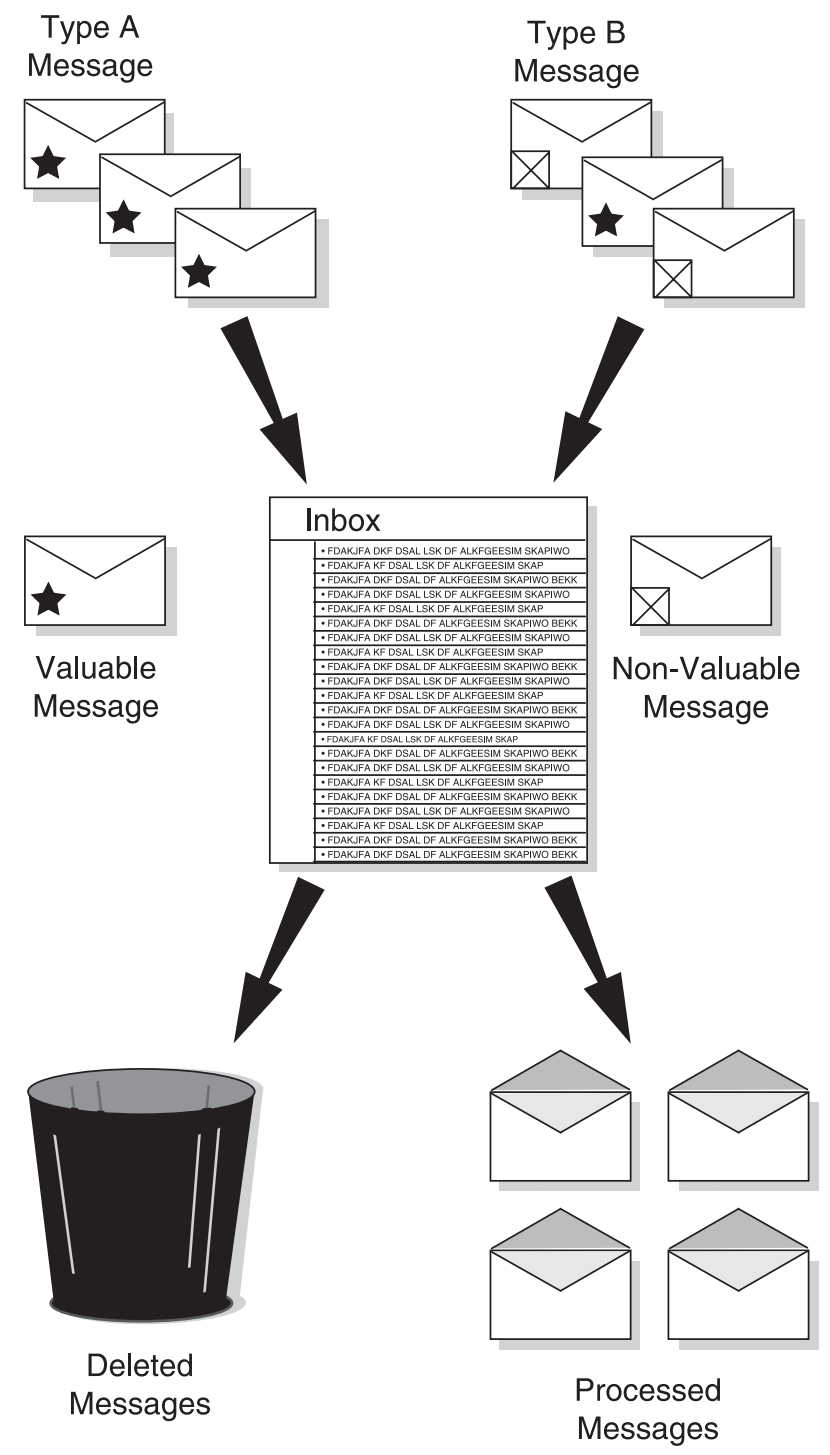

Published online in Wiley InterScience (www.interscience.wiley.com) DOI: 10.1002/sdr 
Each period, a flow of messages $m_{i}$ is added to the backlog, a mix of A and B messages. The backlog can be reduced either because messages are processed or because they are deleted. The two corresponding rates are $m_{o}$ and $m_{d}$. The message backlog is described by the equation

$$
(\mathrm{d} / \mathrm{d} t) M=m_{i}-m_{o}-m_{d}
$$

Figure 6 shows the stock and flow representation of Eq. 1. Below we explain these three rates.

We denote the flow of type-A messages as $a$. The flow of type-B messages is $b$. We assume that all A messages are valuable to the recipients. However, recipients only value a fraction $\rho$ of B messages. Hence, the flow of valuable B messages is $b_{V}=\rho b$. Then, among all messages, the flow of valuable messages is $m_{v}=a+b_{v}$. Since we assume that all A messages are valuable, the flow of nonvaluable messages that arrive to the organization consists only of the flow of non-valuable B messages, that is, $m_{n}=b-b_{v}$.

Incoming messages are analyzed by a filter. For simplicity, we assume that messages identified by the filter as non-valuable are discarded. Ideally, all A messages should be properly identified and delivered to the inboxes and only valuable B messages should be delivered. However, the filter may discard mistakenly some valuable messages (false positive) and may let some nonvaluable messages through (false negative). We denote the percent of false positives as $\eta_{1}$ and percent of false negatives as $\eta_{2}$. An ideal filter makes no errors, that is, $\eta_{1}=\eta_{2}=0$. No filtering implies misspecification rates of $\eta_{1}=0$ and $\eta_{2}=1$. The worst filter has misspecification rates of $\eta_{1}=\eta_{2}=1$.

The filter recognizes $m_{v}^{f}=\left(1-\eta_{1}\right) m_{v}$ of valuable messages as valuable, and

$$
m_{n}^{f}=\eta_{2} m_{n}
$$

of non-valuable messages are also identified as valuable (i.e., false negatives). The message addition rate is the flow of messages that pass through the filter and are added to the backlog, $m_{i}=m_{v}^{f}+m_{n}^{f}$.

Since demand for attention and demand for time are two closely related concepts, we represent attention in units of time. Note that it is possible, if desired, to be more specific in representing attention (Pashler, 1998), but thinking of attention as time will suffice for this analysis. We assume that recipients spend some limited combined budget of time $T=R T_{R}$ on processing messages, where $R$ is the number of recipients and $T_{R}$ is the average time that a recipient spends processing messages each day. The message processing rate, $m_{o}$, depends on the time available $T$ and the average time it takes to process a message, $\tau$. The rate $m_{o}$ does not exceed the typical volume processed every day, $M / \gamma$, where $\gamma$ is the normal response delay. We assume $\gamma$ is constant. Then, the message processing rate is

$$
m_{o}=\min (T / \tau, M / \gamma)
$$




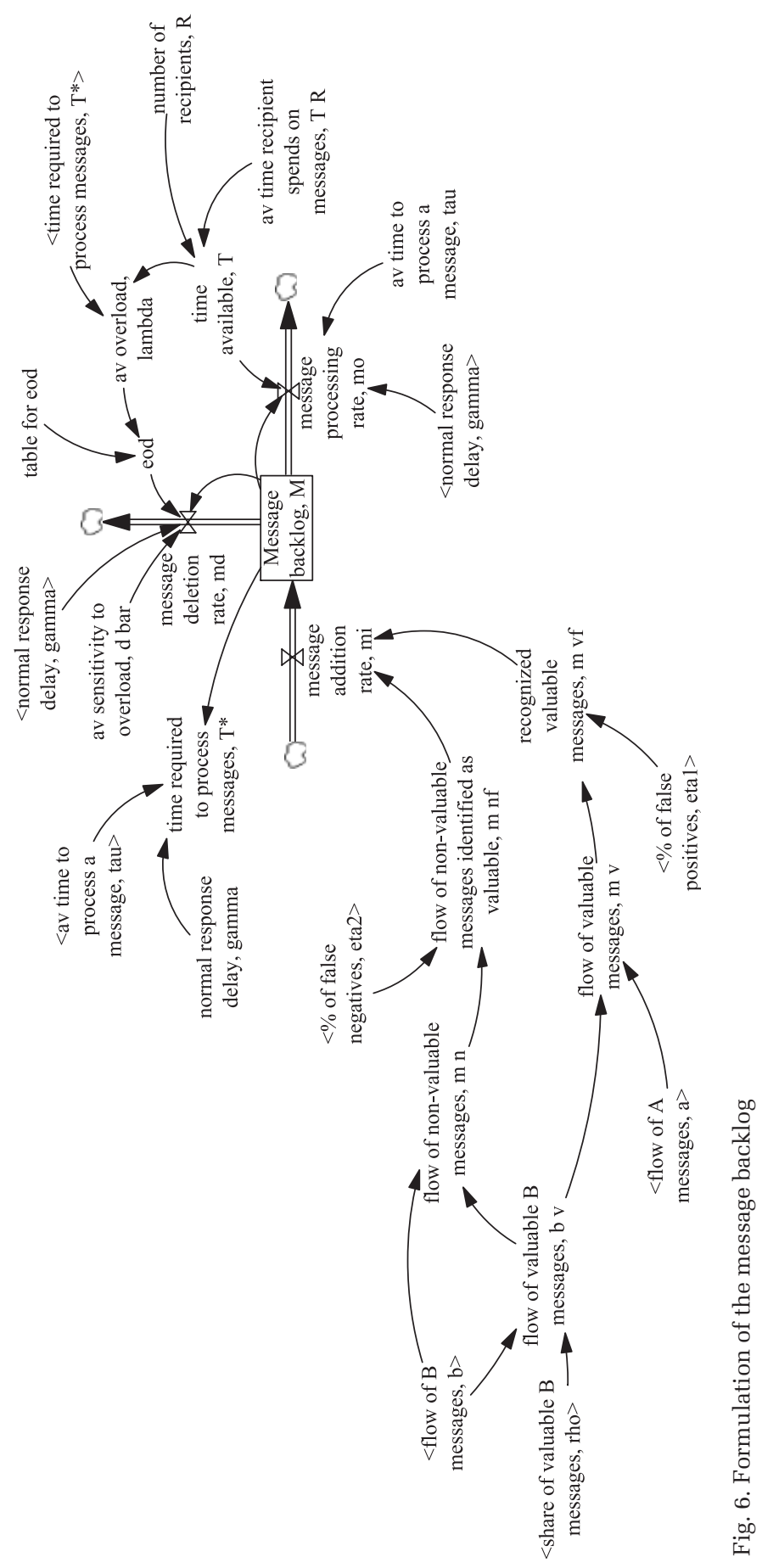

Published online in Wiley InterScience (www.interscience.wiley.com) DOI: 10.1002/sdr 
Note that this formulation depends on two assumptions. First, it assumes perfect intertemporal mixing; that is, no FIFO rule is followed in answering messages. Second, it assumes that a recipient has no way of determining whether or not a particular message is valuable until he or she processes it.

To process $M / \gamma$ messages, recipients require time $T^{*}$, proportional to the average time it takes to process a typical message $\tau$ :

$$
T^{*}=(M / \gamma) \tau
$$

We define average overload among the recipient population as the ratio of the time required to complete a task to the actual time available for the task: $\lambda=T^{*} / T$. Here the task is to process $M / \gamma$ messages. When sufficient time is available to process messages, there is no overload and $\lambda$ falls in the range between zero and one. A value $\lambda>1$ implies that recipients experience overload.

A survey found that, once there is an excessive amount of e-mail, people tend to delete e-mail without reading it (EmailLabs, 2006). We capture this finding by specifying that as the average overload $\lambda$ increases, so does the number of messages that are deleted without being processed. We assume that the average message deletion rate $m_{d}$ is a smooth S-shaped function of the average overload $\lambda$. We describe the message deletion rate as

$$
m_{d}=e o d \cdot \bar{d} M / \gamma
$$

The effect of overload on deletion, eod, is an S-shaped curve (Figure 7). The parameter $\bar{d}$ determines the magnitude of the deletion rate. The ratio $M / \gamma$ acts as a first-order control.

Backlog attributes

The model tracks message backlog attributes required for the analysis of the attention bond mechanism. The attributes of interest are the average fraction of

Fig. 7. Graphical table function for eod

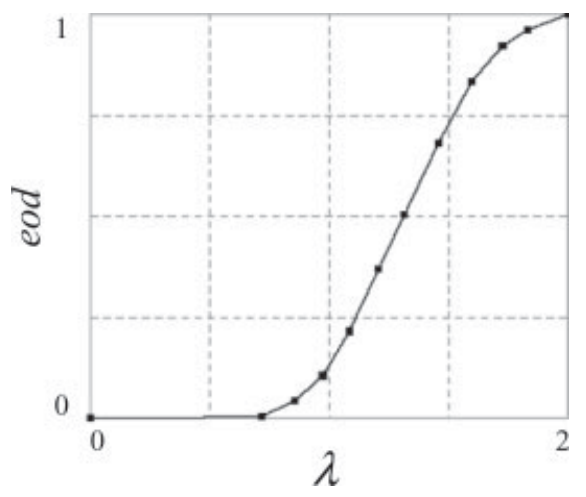

Published online in Wiley InterScience (www.interscience.wiley.com) DOI: 10.1002/sdr 


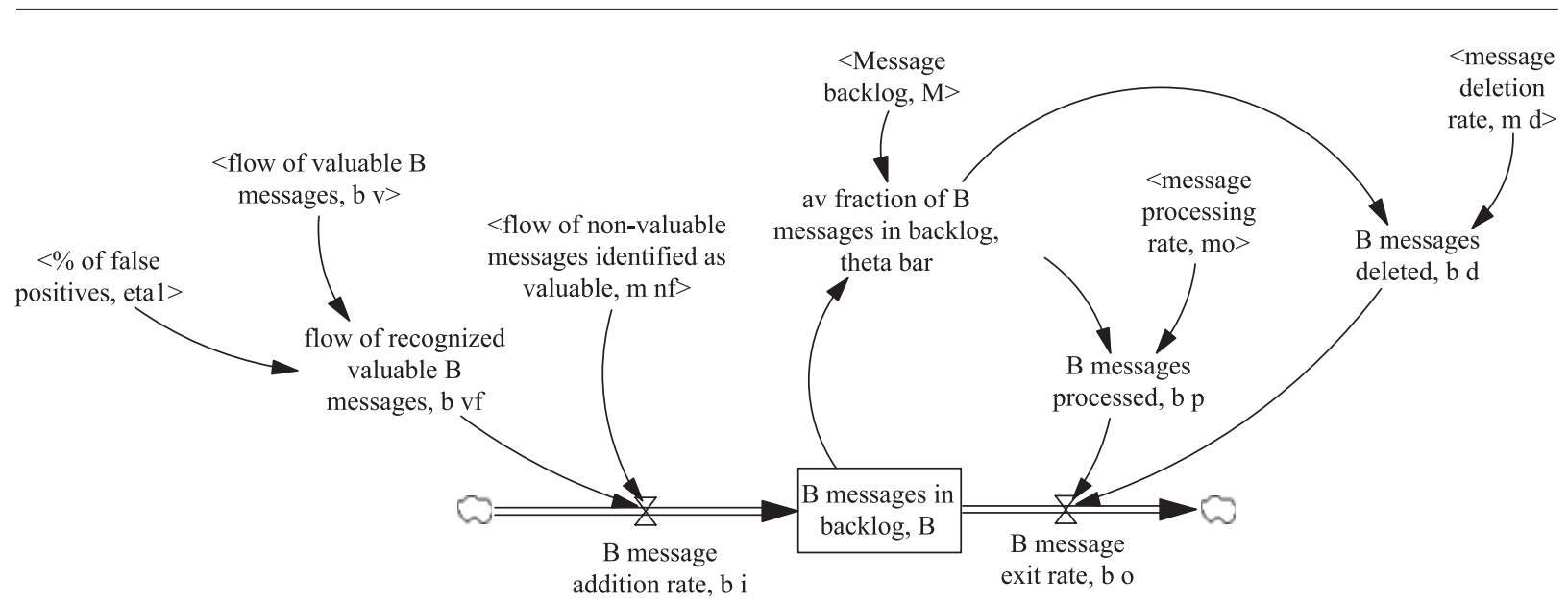

Fig. 8. The average fraction of B messages in the backlog model section

B messages and the average fraction of valuable B messages in the backlog. To compute the attributes, we use the coflow formulation described below.

Figure 8 shows the model section that calculates the average fraction of $B$ messages in the backlog. The number of B messages in the backlog is

$$
\frac{\mathrm{d} B}{\mathrm{~d} t}=b_{i}-b_{o}
$$

The $\mathrm{B}$ message addition rate $b_{i}$ is equal to the number of type- $\mathrm{B}$ messages added to the backlog $M$. The $\mathrm{B}$ message exit rate $b_{o}$ describes the removal of $\mathrm{B}$ messages from the backlog $M$, either through processing or deletion. The average fraction of $\mathrm{B}$ messages in the backlog is

$$
\bar{\theta}=\frac{B}{M}
$$

The rate $b_{i}$ is equal to $b_{i}=b_{V}^{f}+m_{n}^{f}$, where

$$
b_{V}^{f}=\left(1-\eta_{1}\right) b_{v}
$$

is the flow of valuable B messages that pass the filter and are added to the backlog, and $m_{n}^{f}$ is the flow of non-valuable B messages that pass through the filter (Eq. 2). The B message exit rate is defined as

$$
b_{o}=b_{p}+b_{d}
$$

This rate is equal to the sum of $\mathrm{B}$ messages processed, $b_{p}=m_{o} \bar{\theta}$, and $\mathrm{B}$ messages deleted, $b_{d}=m_{d} \bar{\theta}$, from the message backlog $M$. 


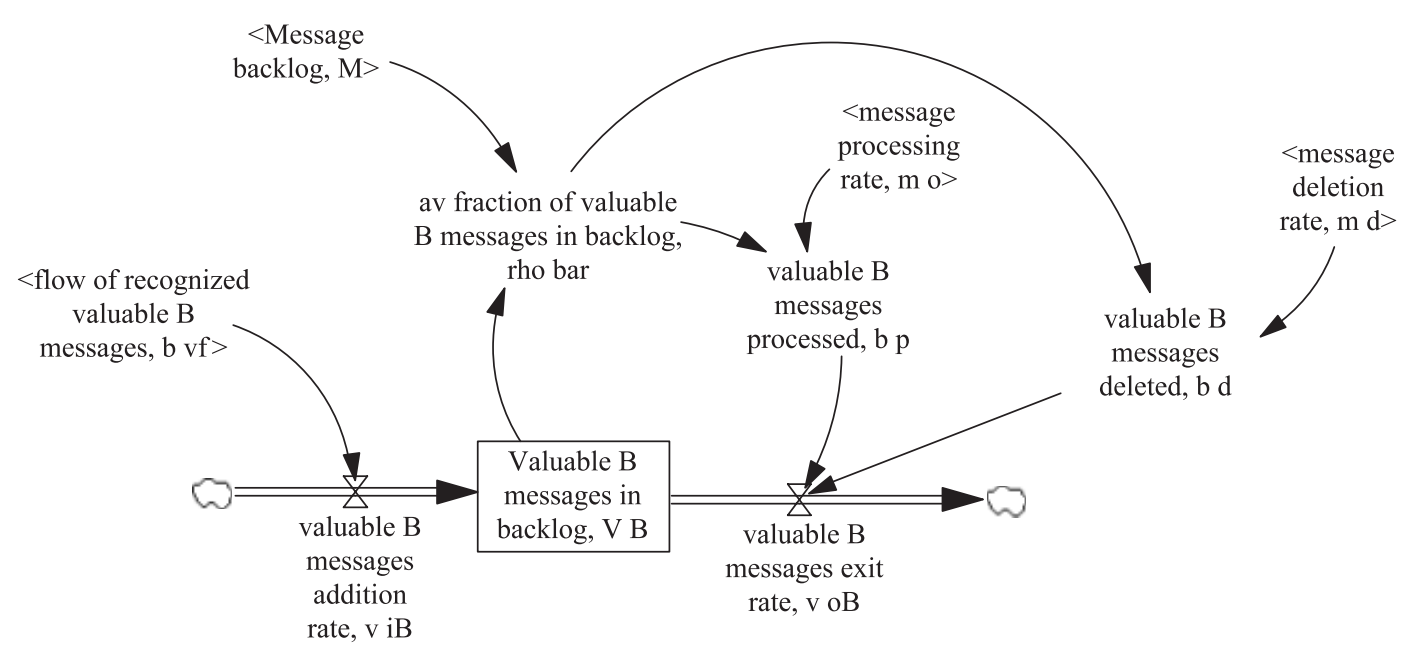

Fig. 9. The average fraction of valuable B messages in the backlog model section

Figure 9 shows the model section that computes the average fraction of valuable B messages in the backlog. The number of valuable type-B messages in the backlog is

$$
\frac{\mathrm{d} V^{B}}{\mathrm{~d} t}=v_{i}^{B}-V_{o}^{B}
$$

Here, $v_{i}^{B}$ is the valuable $\mathrm{B}$ messages addition rate that accounts only for valuable type-B messages added to backlog $M$. The valuable B messages exit rate $v_{o}^{B}$ equals the number of valuable type-B messages that are either processed or deleted each day, and $\bar{\rho}$ is the average fraction of valuable type-B messages in the backlog:

$$
\bar{\rho}=\frac{V^{B}}{M}
$$

We also define the rates $v_{i}^{B}=b_{v}^{f}$ (Eq. 8) and $v_{o}^{B}=b_{p}^{v}+b_{d}^{v}$. Here,

$$
b_{p}^{v}=m_{o} \bar{\rho}
$$

are the valuable B messages processed per day, and $b_{d}^{v}=m_{d} \bar{\rho}$ are the valuable B messages deleted per day.

\section{Sponsors}

Sponsors financially support communication of broadcasters B. In Figure 10, communication funding, $S$, is equal to the difference between the new budget 
Fig. 10. The Sponsors model sector

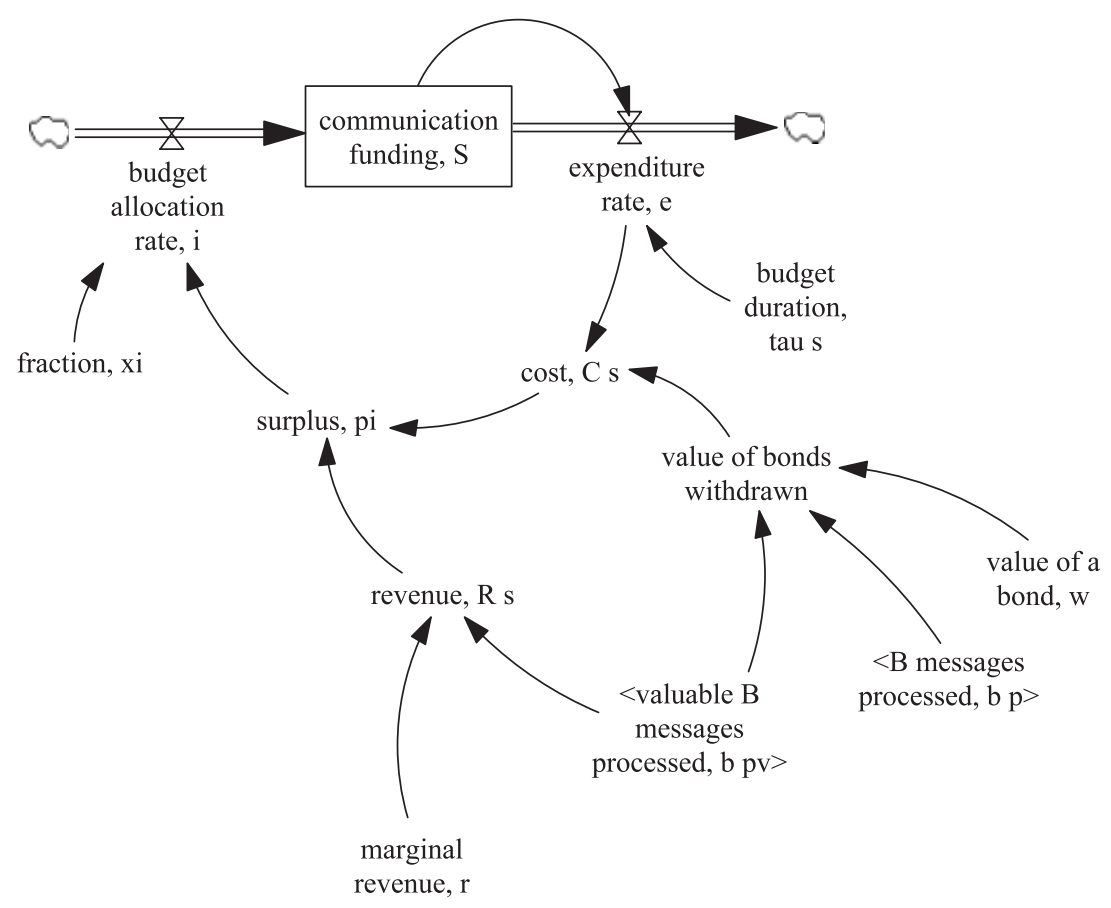

allocations $i$ and the expenditure on broadcasting, $e$, which is paid to broadcasters B:

$$
(\mathrm{d} / \mathrm{d} t) S=i-e
$$

Once allocated, the budget is spent over a budget duration $\tau_{S}$ :

$$
e=S / \tau_{S}
$$

Assuming that sponsors have no fixed cost, they earn a surplus $\pi=R_{S}-C_{S}$, where $R_{S}$ is the sponsors' revenue and $C_{S}$ is the sponsors' cost. We model the expected per-message sponsor revenue by assuming that each time recipients process a valuable type-B message sponsors receive a marginal revenue $r$. Assuming a constant marginal revenue, sponsors receive revenue $R_{S}=r b_{p}^{v}$, where $b_{p}^{v}$ are the responses that sponsors receive per day, equal to the valuable $B$ messages processed per day.

Although companies use a variety of approaches to budget for commercial email (EmailLabs, 2006, p. 26), we assume for simplicity that sponsors allocate a fraction $\xi$ of surplus to broadcasting. Considering that allocations must be non-negative, the budget allocation rate is

$$
i=\max (\xi \pi, 0)
$$


Fig. 11. The

Broadcasters A model sector

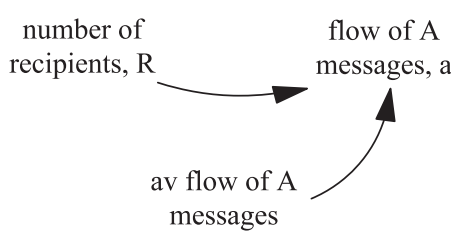

If an attention bond system is applied, then the sponsor must pay some fixed amount to recipients each time a non-valuable message is processed. To accommodate these payments, sponsor cost includes the value of warrants withdrawn, which is a product of the value of a bond, $\omega$, and the number of bonds cashed. The bonds cashed is the difference between type-B messages processed, $b_{p}$, and valuable type-B messages processed, $b_{p}^{v}$. Hence, the withdrawn bonds value is $\omega\left(b_{p}-b_{p}^{v}\right)$. The resulting cost is the sum of the expenditure on commercial e-mail and the withdrawn bonds value:

$$
C_{S}=e+\omega\left(b_{p}-b_{p}^{v}\right)
$$

Setting $\omega=0$ is equivalent to a regime without a bond system.

\section{Broadcasters}

There are two types of broadcasters: A and B. Broadcasters A (Figure 11) send a flow $a$ of type-A messages on their own behalf. We set $a=a_{R} R$, where $a_{R}$ is the average flow of A messages per recipient per day and $R$ is the number of recipients.

Figure 12 shows the Broadcasters B model sector. Broadcasters B send type$\mathrm{B}$ messages, but only if sponsors pay. The flow of B messages is determined by the sponsor expenditure rate, $e$, and is inversely proportional to the base price of sending a $\mathrm{B}$ message $p_{b}$ :

$$
b=e / p_{b}
$$

Fig. 12. The Broadcasters B model sector

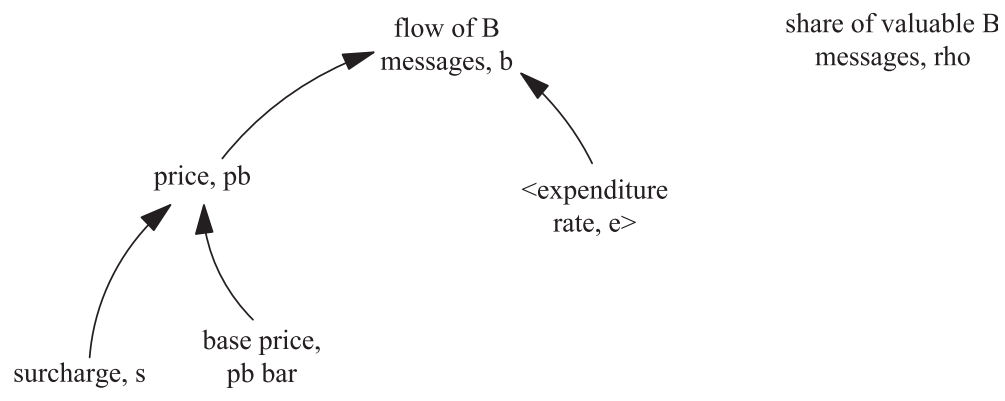

Published online in Wiley InterScience (www.interscience.wiley.com) DOI: 10.1002/sdr 
Fig. 13. Receiver operating characteristic (ROC) curve and its shifts. Parameter $\eta_{1}$ denotes the percent of false positives and $\eta_{2}$ is the percent of false negatives (adapted from Cormack, 2006)

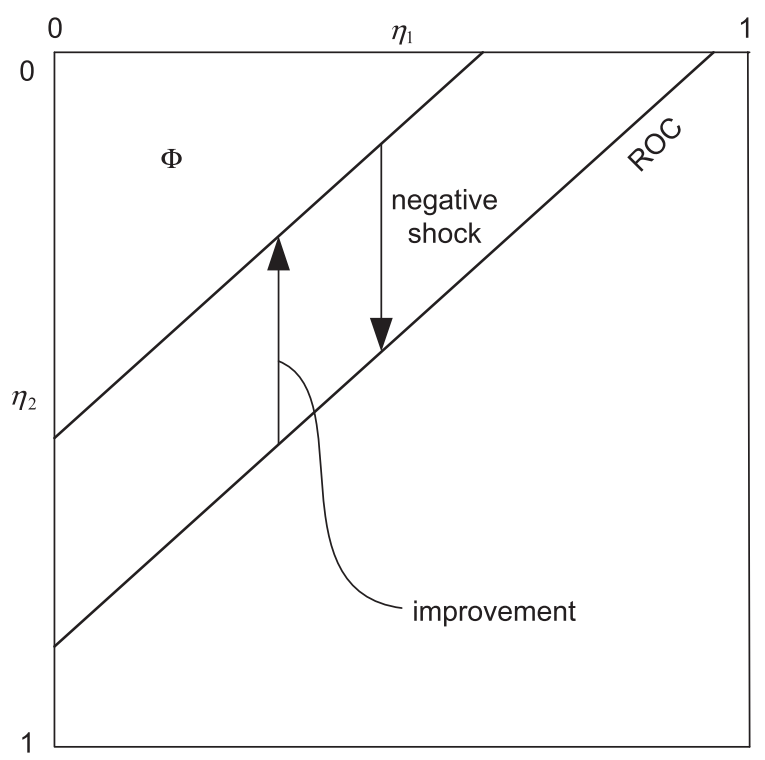

Price is

$$
p_{b}=\bar{p}_{b}+s
$$

where $\bar{p}_{b}$ is the base price per B message and $s$ is a surcharge for each type-B message. Note that the surcharges do not apply to Broadcasters A.

Broadcasters B also set the share of valuable B messages, $\rho$, in the flow of B messages. Broadcasters can improve $\rho$ through better targeting and customization, for example, but $\rho$ is fixed in this model.

\section{IT Company}

The IT Company provides a communication filter. Its purpose is to block nonvaluable messages.

With all filters, there is a tradeoff between false-positive and false-negative percentages (Cormack, 2006). It is possible to achieve zero false positives, but this would imply higher false negatives. For example, no filtering at all produces zero false positives at the expense of 100 percent false negatives. This tradeoff can be graphically shown as a Receiver Operating Characteristic (ROC) curve (Figure 13). Better filters correspond to higher curves. Point $(0,0)$ corresponds to an ideal filter. The worst filter situation is described by point $(1,1)$, which is actually worse than having no filter. The point equivalent to the absence of a filter is $(0,1)$. A convenient summary measure of a filter's performance is 1 - ROCA, where ROCA is the area under the ROC curve (Cormack, 2006). The 1 - ROCA is a number between 0 and 1 , which measures the overall 
Fig. 14. The IT

Company model sector

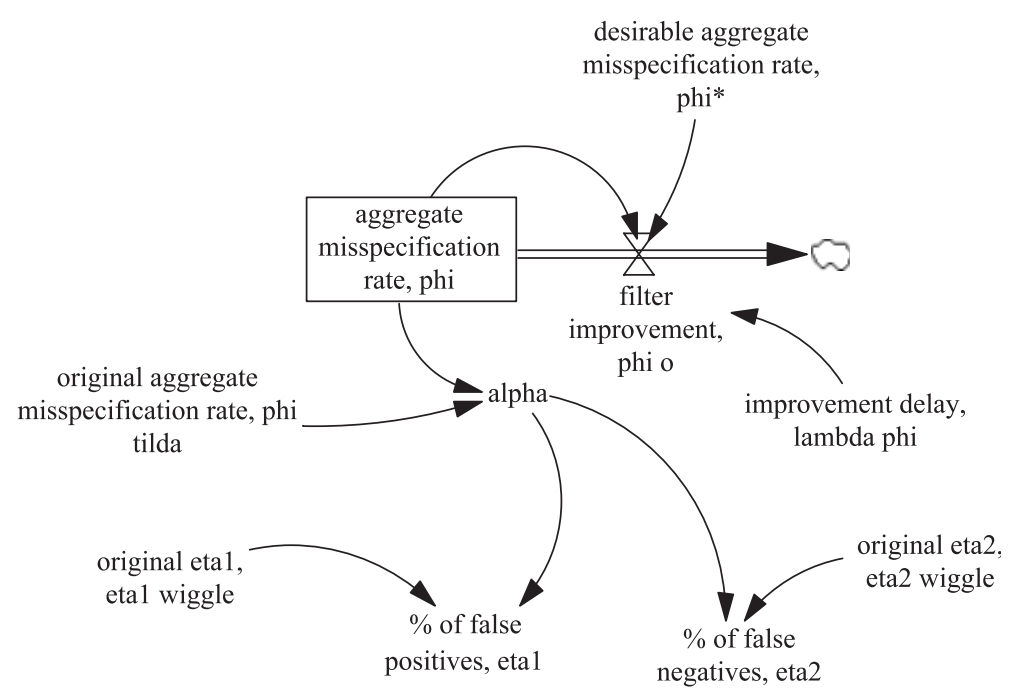

misspecification percentage. The ideal filter corresponds to $1-$ ROCA $=0$, and the worst filter means $1-$ ROCA $=1$.

Figure 14 shows the stock and flow diagram of the IT Company. For notational convenience, we will refer to 1 - ROCA as $\Phi$ (Figure 13). Filter improvement $\phi_{o}$ decreases the misspecification rate $\Phi$ :

$$
(\mathrm{d} / \mathrm{d} t) \Phi=-\phi_{o}
$$

Filter improvements are driven by the gap between the desirable aggregate misspecification rate $\Phi^{*}$ and the current state of the filter $\Phi$ :

$$
\phi_{o}=\left(\Phi-\Phi^{*}\right) / \lambda_{\phi}
$$

where $\lambda_{\phi}$ is the filter improvement delay. Recipients have some desired $\Phi^{*}$ based on the balance of effort to process e-mail versus the cost of a better filtering tool. We set $\Phi^{*}$ exogenously.

Suppose the ROC line in Figure 13 is above the 45-degree line, so that $0 \leq \Phi<0.5$. As the filtering technology improves, $\Phi$ changes by a certain ratio $\alpha=\Phi / \tilde{\Phi}$, where $\tilde{\Phi}$ is the original aggregate misspecification rate. Even though a simplification, we also assume that the tradeoff between false positives and false negatives is constant over time. This implies that the slope of the ROC curve remains constant, which allows using properties of similar triangles to find $\eta_{1}$ and $\eta_{2}$ as the ROC curve shifts. Rates $\eta_{1}$ and $\eta_{2}$ change by the factor $\sqrt{\alpha}$ from their original values $\tilde{\eta}_{1}$ and $\tilde{\eta}_{2}$ :

$$
\begin{aligned}
& \eta_{1}=\tilde{\eta}_{1} \sqrt{\alpha} \\
& \eta_{2}=\tilde{\eta}_{2} \sqrt{\alpha}
\end{aligned}
$$


Table 1. Validation tests
Test

Increasing the number of recipients $R$ also increases e-mail backlog, but it should not change the average overload in the steady state

2 In the presence of filtering, $\bar{\rho}$ should be greater than $\rho$

3 In the situation equivalent to the absence of filtering, i.e., $\eta_{1}=0$ and $\eta_{2}=1$, steady-state values of the average fraction of valuable type-B messages in the backlog, $\bar{\rho}$, should be equal to the share of valuable messages among type-B messages, $\rho$

4 Steady-state values must satisfy these logical conditions: $V^{B}<M$ and $V^{B} \leq B$

$5 \quad$ If $\Phi$ drops by $\alpha$ percent, then $\eta_{1}$ and $\eta_{2}$ drop by $\sqrt{\alpha}$ percent

6 Increasing the marginal revenue $r$ should increase sponsor surplus, and therefore increase, at least initially, the flow of B messages

$7 \quad$ Model behavior must be robust to variations in $\bar{d}$

8 Completely eliminating type-B messages reduces both the e-mail backlog and the attention overload

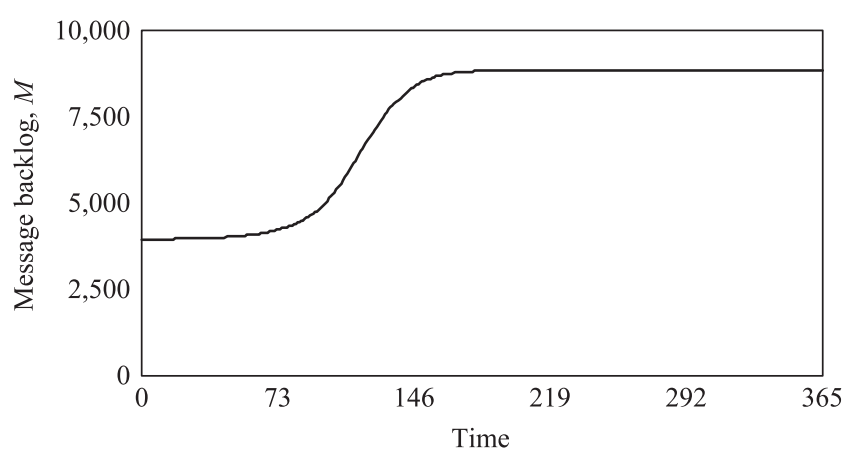

(a)

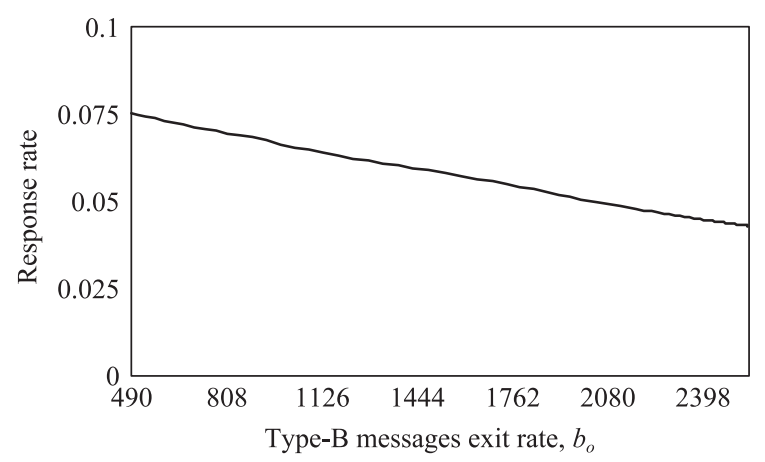

(b)

Fig. 15. Growth run

\section{Validation of the model}

We validated the model by performing a series of simulations that tested for behavioral anomalies, response to parameter changes and behavior under extreme conditions. The model passed each of our tests, which we summarize in Table 1. We also performed a units check.

For one of the runs, we assigned low initial values for stocks message backlog $M$, valuable B messages in backlog $V^{B}$, B messages in backlog $B$, and communication funding $S$. The S-shaped trajectory for $M$ in Figure 15(a) is expected for our model, which is a variant of the limits-to-growth generic structure (Figure 3). The run in Figure 15(a) also confirms that spam production can increase-the Feared scenario in Figure 1. 
We used the same growth run to test the simulated response rate. Since the message backlog formulation (Eq. 1) assumes perfect mixing, we can only approximate the response rate as a ratio of all processed valuable $\mathrm{B}$ messages $\left(b_{p}^{v}\right.$, Eq. 12) to all type-B messages processed and deleted (that is exit rate $b_{o}$, Eq. 9):

$$
\text { response rate }=\frac{b_{p}^{v}}{b_{o}}
$$

The simulated response rate (Figure 15(b)) declines as the flow of B messages increases, which corresponds to the reference mode on Figure 2(a).

\section{Policies}

We start policy experiments from a steady state. Parameter values and steadystate values for stocks are listed in the Appendix.

\section{Better filter}

Filtering has been the most widely deployed tool for screening the unwanted commercial messages in e-mail. The research to improve the ability of filters to correctly classify spam is ongoing (Goodman et al., 2007), which raises the question: Do better filters affect the aggregate flow of spam? We model an improvement in the filter technology by lowering the desirable aggregate misspecification rate $\Phi^{*}$ at time 30 from 0.1 to 0.05 , which translates into lower misspecification rates $\eta_{1}$ and $\eta_{2}$ (Eq. 21). The effect of a better filtering effort can be traced in Figure 3.

The improved filter blocks more messages (Figure 16(a)). The blocking rate equals the flow of incoming messages less the message addition rate, $m_{b}=a+b$ $-m_{i}$. The rise in the simulated blocking rate corresponds to the reference mode in Figure 2(c). Note that because of the improvement delay $\lambda_{\phi}$ (Eq. 20), the simulated trajectory shows a gradual increase, rather than the step-wise increase of the reference mode.

An improved filter lowers the flow of non-valuable messages added to the backlog. Therefore, the average fraction of valuable B messages in the backlog increases (Figure 16(c)). Having more valuable type-B messages in the backlog improves revenue and surplus for sponsors (Figures 3 and 16(e)). In turn, greater surplus increases communication funding (Figure 3). Given a constant price for a marketing message, greater communication funding results in a greater flow of type-B messages (Figures 3 and 16(f)). The growth in the flow of $\mathrm{B}$ messages leads to the increase in the flow of $\mathrm{B}$ messages that pass the filter and are added to the backlog (Figure 16(b)). This implies that the reference mode of Figure 2(b) does occur, but the decline in delivered electronic ads is 


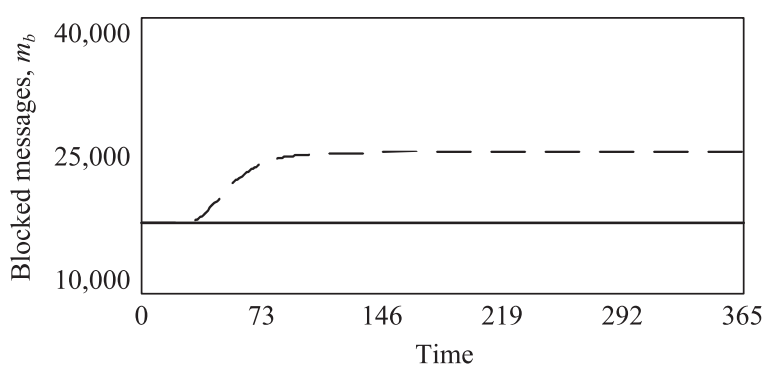

(a)

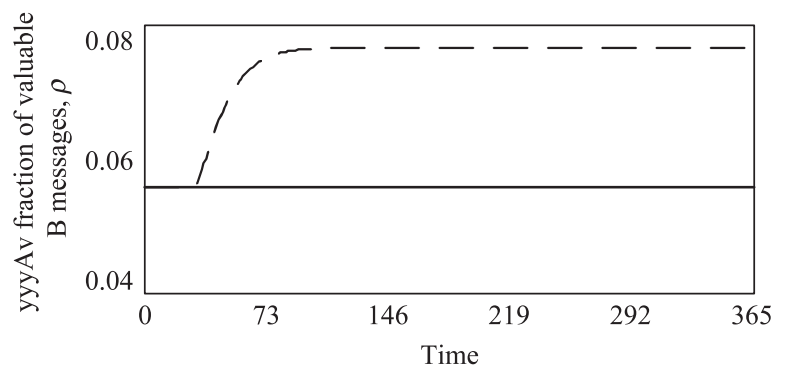

(c)

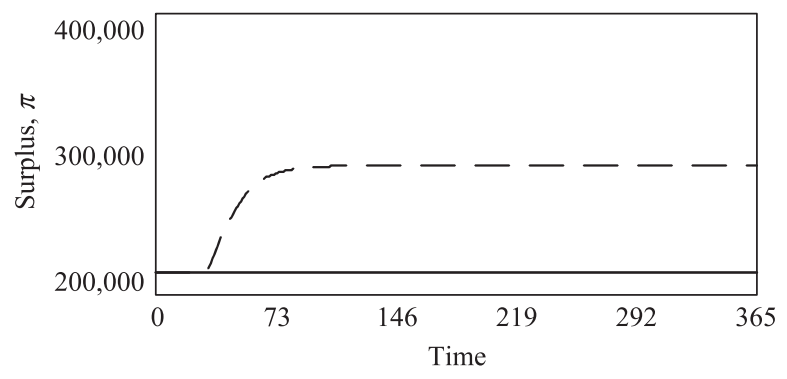

(e)

Regular filter

Improved filter ----

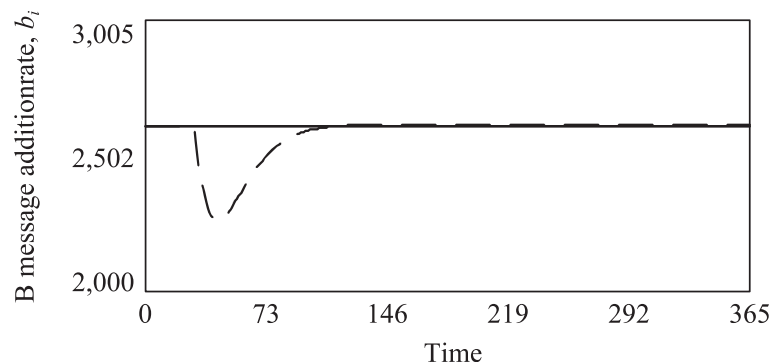

(b)

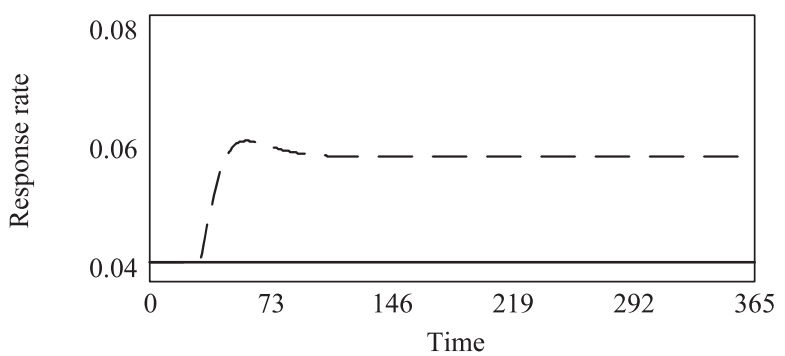

(d)

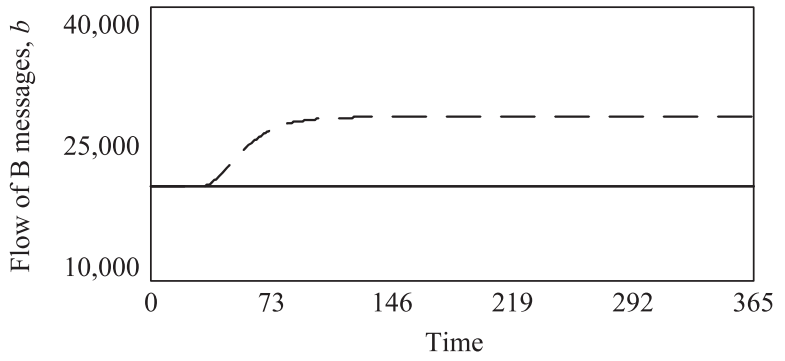

(f)

Regular filter

Improved filter -----

Fig. 16. Improvement of the filter at time 30. Trajectories for the experiment are compared to the base run: (a) improved filter blocks more messages; (b) improved filter briefly reduces the flow of marketing messages into an inbox; (c) fewer non-valuable type-B messages pass the filter; (d) with improved filter, response rates increase; (e) sponsors receive better surplus; (f) surplus leads to greater aggreate flow of spam

not likely to last long. Better filtering improves the response rate (Figure 16(d)) and triggers the reinforcing Spam Growth loop (Figure 3), which drives the spam production to a higher level than before.

An intuitive explanation for this result can be given with the help of Figure 3. An improvement of a filter, say, through better calibration and training, reduces filtering errors. Fewer errors means that the filter blocks messages 
Fig. 17. Introduction of a surcharge at time 30. In each run, the surcharge is a percent of the base price of broadcasting $\bar{p}_{b}$ : (a) surcharge reduces type-B flow; (b) surcharge reduces sponsor surplus; (c) surcharge leads to less funding; (d) surcharge lowers overload, please see next page for (c) and (d)

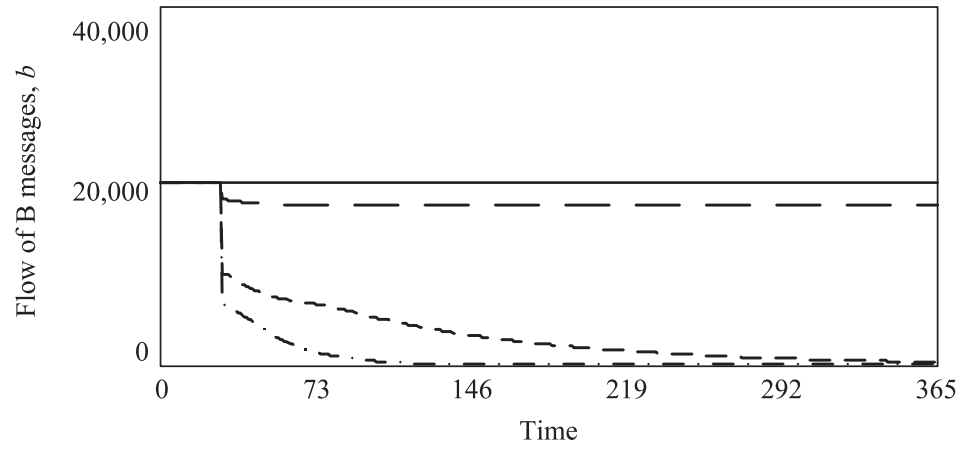

(a)

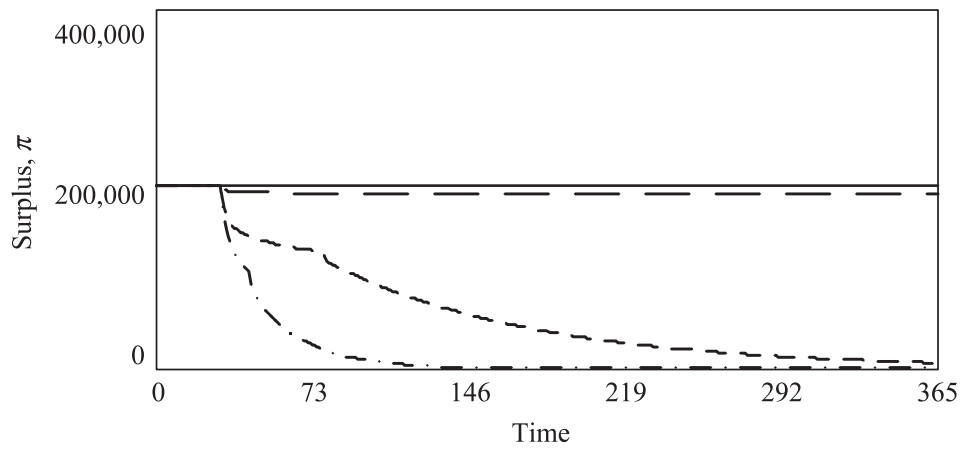

(b)

\section{Base run}

Surcharge $10 \%$

Surcharge $100 \% \quad--------$

Surcharge $200 \% \quad-\cdot-\cdot-\cdot \cdot-\cdot-\cdot \cdot \cdot$

that are of no interest to recipients but allows valuable messages through. In effect, a good filter does the work of targeting for spammers. Thinking in terms of the causal loop diagram in Figure 3, improved targeting and filtering both improve the fraction of valuable marketing messages in the backlog. Therefore, both improved targeting and improved filtering are likely to lead to increased revenue for spammers and an increase in the flow of marketing messages.

\section{Introduction of surcharge}

To test the effect of a surcharge scheme on sponsored communication, we conduct a series of simulations by adding a surcharge at time 30 to the base 
Fig. 17. (Continued)

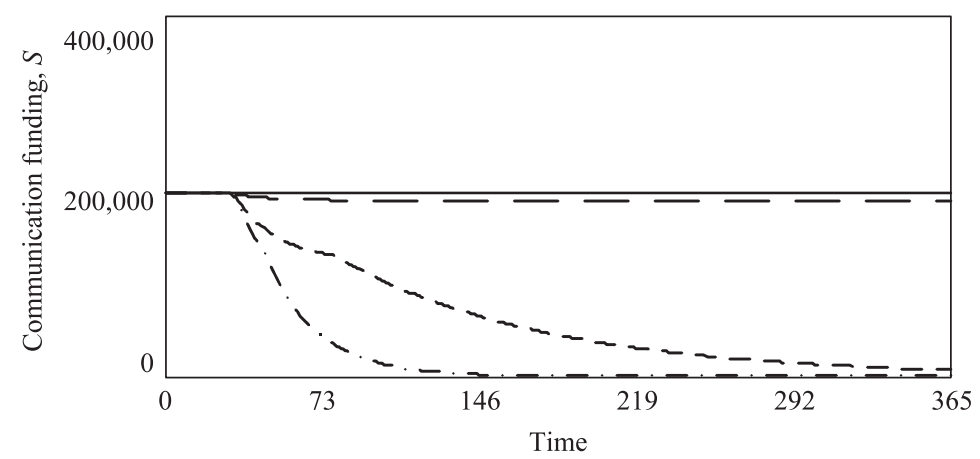

(c)

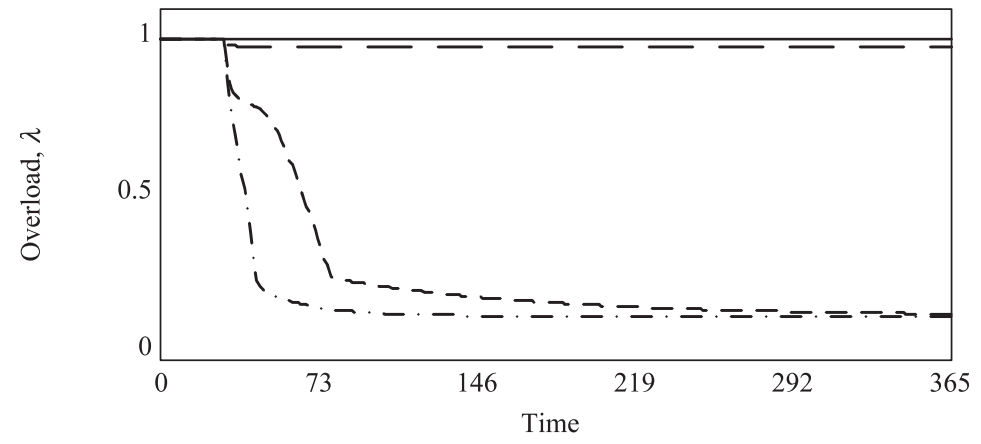

(d)

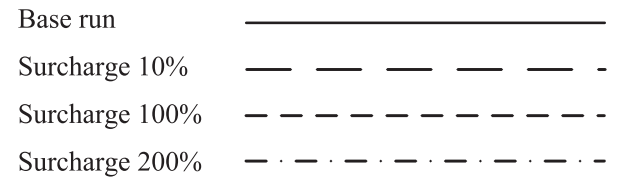

price (Eq. 18). The surcharge is a percent of the base price. The introduction of a surcharge is modeled with the step function.

Figure 3 (top left) shows the causal chain of the surcharge and Figure 17(a) shows the corresponding drop in the production of type-B messages. The surcharge lowers revenue for sponsors (Figure 3), surplus (Figure 17(b)) and communication funding (Figure 17(c)). The resulting reduction in the message backlog implies lower than before overload (Figures 3 and 17(d)). These experiments suggest that a sufficiently high surcharge can lead to a market failure, so that for-profit Broadcasters B stop sending messages. This is not a desired outcome (Figure 1). 
Fig. 18. Introduction of the attention bond at time 30 . Bond values are percentages of the marginal revenue $r$ : (a) bond system cuts into sponsor surplus; (b) bonds lower communication funding; (c) bonds lower the flow of marketing messages; (d) bonds lower information overload, please see next page for (c) and (d).

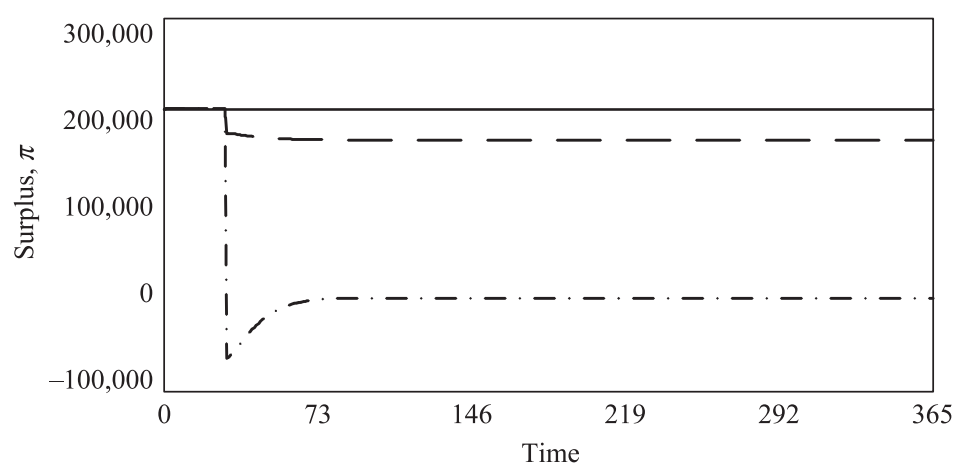

(a)

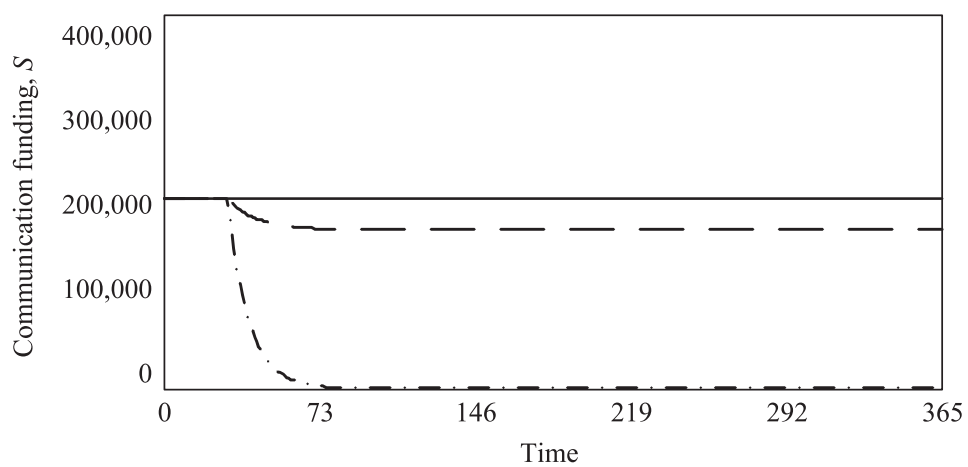

(b)

Base run

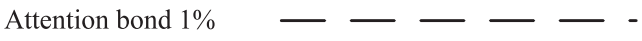

Attention bond $10 \% \quad-\cdot-\cdot-\cdot \cdot-\cdot \cdot-\cdot$

\section{Adoption of an attention bond mechanism}

An attention bond mechanism is simulated by changing parameter $\omega$ (Eq. 16) from zero to a positive value. We use the step function to simulate this change. We run two simulations, assuming initially that the value of a bond is 1 percent of the sponsor's marginal revenue $r$, and then setting the value of the attention bond to 10 percent of $r$. When the value of a bond, $\omega$, increases, it immediately lowers sponsor surplus (Figure 3), communication funding and marketing messages. The message backlog is reduced since fewer messages arrive now than before. Figure 18 shows the corresponding trajectories. Excessively high bond payments can leave sponsors with losses (Figure 18(a)), which may lead to the elimination of communication funding (Figure 18(b)) and suspension of all type-B broadcasting (the case of a 10 percent bond on Figure 18(c)). From a societal standpoint, eliminating all marketing communication is not a 
Fig. 18. (Continued)

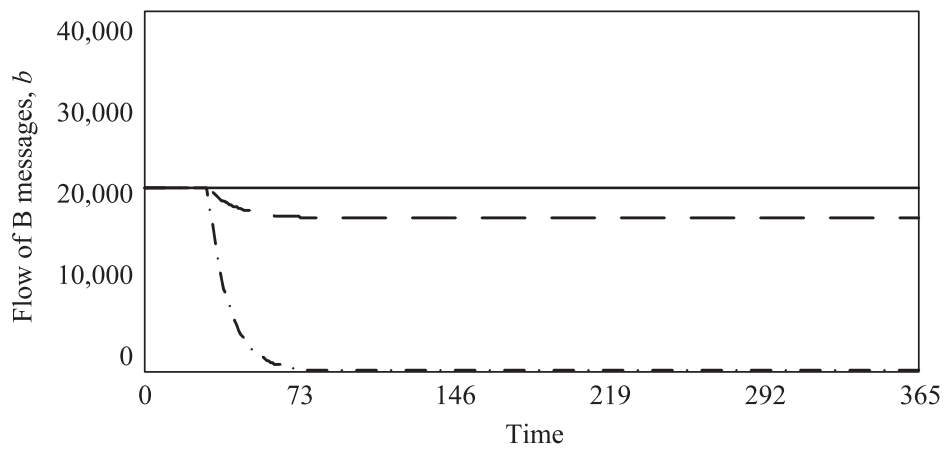

(c)

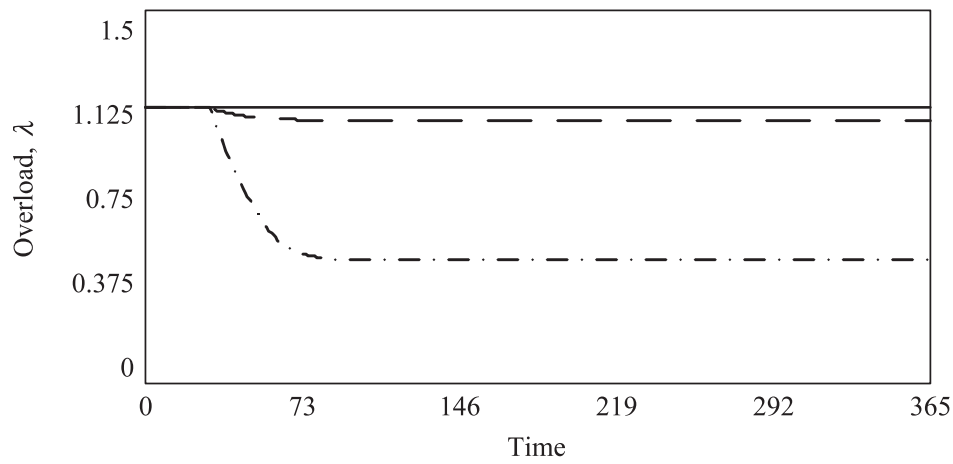

(d)

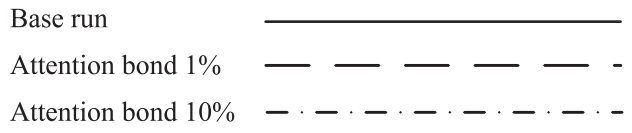

desired outcome (Figure 1). Simulations (Figure 19) reproduce the reference mode in Figure 2(d).

\section{Conclusion}

This paper presents a generic model of message-based communication, with recipients that have a limited information-processing capacity. Profit-seeking broadcasters and sponsors exploit the freely available but limited attention endowment of recipients. We examine the likely effects of filtering, surcharges and the introduction of an attention bond. We assume that every recipient has access to filtering and that the surcharge and attention bond policies are universal, i.e., for-profit broadcasters and sponsors cannot avoid them. 
Fig. 19. An attention bond improves the response rate

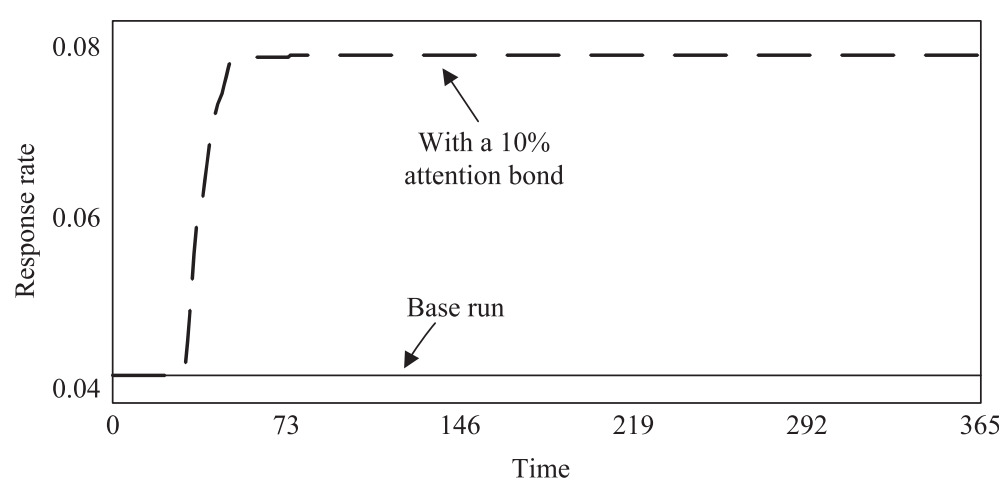

Our analysis reveals the following findings:

1. A commercial communication system that depends on the attention of recipients (as, for example, spam) may be described by a causal structure that is an extension of the limits-to-growth generic system archetype.

2. Filtering, while effective at blocking unwanted messages, may encourage the production of commercial messages. In other words, the widespread deployment of filters and their improvement can increase, rather than decrease, the amount of spam transmitted on the Internet and other communication platforms.

3. By increasing the cost of communication, surcharges and attention bonds can depress and even completely eliminate commercial communication. This finding implies that poorly designed market-based mechanisms may lead to market failure, not a socially desirable outcome.

The above findings have significant implications for the management of unwanted commercial messages at the organizational and global levels. First, marketers, managers and policy makers must recognize the importance of mitigating excessive external demands on the limited attention endowments of employees and customers. E-mail has, within just a few years, advanced from an arcane fringe technology to the dominant means of business communication within developed economies. When technology changes quickly, there is a risk that management may be slow to appreciate the unintended negative externalities associated with its deployment. Second, management must proceed cautiously in embracing solutions that have not been modeled within a realistic dynamic framework that accounts for the feedback effects and incentives of the various players, whose interactions determine likely outcomes.

A third observation, pertinent to the management of commercial messages, is that the optimal solution to the communications-related attention overload 
may not be achievable by firms acting independently. E-mail filters, for example, can mitigate the overload problem locally, within the boundaries of a single firm and, if all other things are held constant, a firm may be made better off by introducing improved filters. But, as finding 2 implies, if all firms do the same, then the global result may be more spam than otherwise and more, rather than less, spam for the (always imperfect) filters to deal with. Each firm's decision to deploy filters has an externality effect on other firms that use email. The ultimate solution to the overload problem may require a cooperative policy introduced globally by e-mail users and the ISPs that offer e-mail services.

Our analysis may be extended in a number of ways. A future model could examine non-universal policies. Such a model would capture situations when, for example, imposing surcharges on U.S.-based spammers merely allows foreign-based spammers to increase production. The current model assumes that the attention allocated by recipients to communication is fixed; a future model could relax this assumption. Future analysis may also investigate nonlinear surcharges and attention bonds.

\section{References}

Anderson SP, De Palma A. 2005. A theory of information overload. http://www. cireq.umontreal.ca/activities/papiers/04-05anderson.pdf [16 September 2008].

Chittenden L, Rettie R. 2003. An evaluation of email marketing and factors affecting response. Journal of Targeting, Measurement and Analysis for Marketing 11(3): 203-218.

Claburn T. 2008. The cybercrime economy. Information Week (9 April). http://www. informationweek.com/blog/main/archives/2008/04/the_cyber_crime.html; jsessionid=50IYFHY1JU4QIQSNDLOSKH0CJUNN2JVN?print=true [27 May 2008].

Cormack GV. 2006. The trec 2005 spam filter evaluation track. Virus Bulletin January: S2.

Dai R, Li K. 2004. Shall we stop all unsolicited email messages? In First Conference on Email and Anti-Spam CEAS 2005, Mountain View, CA, 30-31 July 2004.

Davenport TH, Beck JC. 2001. The Attention Economy: Understanding the New Currency of Business. Harvard Business School Press: Boston, MA.

EmailLabs. 2006. Email marketing statistics and metrics. EmailLabs.com. http:// www.emaillabs.com/resources/resources_statistics.html [6 January 2007].

Fahlman S. 2002. Selling interrupt rights: a way to control unwanted e-mail and telephone calls. IBM Systems Journal 41(4): 759-766.

Fallows D. 2003. Spam: how it is hurting email and degrading life on the Internet. PEW Internet Project, Washington, DC.

Gatti J. 2004a. Digital secures significant spot in marketing mix. Direct Marketing February: 1-2.

Gatti J. 2004b. Study: false positives to cost companies $\$ 419 \mathrm{~m}$ by 2008. Direct Marketing February: 1.

Gatti J. 2004c. Survey: spam thwarting online sales growth. Direct Marketing February: 1.

Goldman E. 2006. A Coasean analysis of marketing. Wisconsin Law Review 2006(4): $1151-1221$. 
Goodman J, Cormack GV, Heckerman D. 2007. Spam and the ongoing battle for the inbox. Communications of the ACM 50(2): 25-33.

Greco GM, Floridi L. 2004. The tragedy of the digital commons. Ethics and Information Technology 6(2): 73-81.

Hansell S. 2003. An unrepentant spammer vows to carry on, within the law. New York Times Online 30 December.

Houston FS, Scott D. 1984. The determinants of advertising page exposure. Journal of Advertising 13(2): 27-34.

Hunter D. 2003. Cyberspace as place and the tragedy of the digital anticommons. California Law Review 91(2): 439-519.

Khong DWK. 2004. An economic analysis of spam law. Erasmus Law and Economics Review 1 (February): 23-45.

Kleiner K. 2008. Happy spamiversary! Spam reaches 30. NewScientist.com (25 April). http://technology.newscientist.com/article/dn13777-happy-spamiversary-spamreaches-30.html [27 May 2008].

Kraut RE, Sunder S, Morris J, Telang R, Filer D, Cronin M. 2002. Markets for attention: will postage for e-mail help? Yale ICF Working Paper No. 02-28.

Loder T, Van Alstyne M and Wash R. 2006. An economic response to unsolicited communication. Advances in Economic Analysis and Policy 6(1): 1-38.

Magill K. 2007. E-mail roi still a stunner, but diminishing: Dma. Direct Magazine (23 October). http://directmag.com/disciplines/email/email_roi_diminishing/ [May 27 2008].

Marshal. 2007. Marshal security threats: Spam, phishing, malware. http://www.marshal.com/ newsimages/trace/Marshal_Trace_Report-Dec_2007.pdf [25 May 2008].

Martin BA, Durme JV, Raulas M, Merisavo M. 2003. Email advertising: exploratory insights from Finland. Journal of Advertising Research 43(3): 293-300.

McFadden D. 2001. The tragedy of the commons. Forbes ASAP 168: 61-62.

McWilliams B. 2005. Spam Kings. O’Reilly: Sebastopol, CA.

Pashler H. 1998. Attention. Psychology Press: Hove, UK.

Pavlov OV, Melville N, Plice R. 2005. Mitigating the tragedy of the digital commons: the problem of unsolicited commercial e-mail. Communications of the Association for Information Systems 16: 73-90.

Rainie L, Fallows D. 2004. The impact of can-spam legislation. Pew Internet Project: Washington, DC.

Regan P. 2002. Privacy as common good in the digital world. Information, Communication and Society 5(3): 382-405.

Return Path. 2006. Bonded sender program white paper. http://www.returnpath.biz/ pdf/bondedsender.pdf [14 March 2006].

Rubenking NJ. 2004. Antispam tools keep up. PC Magazine 23(14): 30-33.

Rudolph HJ. 1947. Attention and Interest Factors in Advertising. Funk \& Wagnalls: New York.

Rust RT, Espinoza F. 2006. How technology advances influence business research and marketing strategy. Journal of Business Research 59: 1072-1078.

Scalet SD. 2003. The great spam supply chain. CIO Magazine (15 March). http:// www.cio.com/article/31787/The_Great_Spam_Supply_Chain [25 May 2008].

Senge PM. 1990. The Fifth Discipline: The Art and Practice of the Learning Organization. Doubleday/Currency: New York.

Starch D. 1966. Measuring Advertising Readership and Results. McGraw-Hill: New York.

Stix G. 2002. Tragedy of the cyber commons. Scientific American 286(4): 36.

Sweet M. 2003. Political e-mail: protected speech or unwelcome spam? Duke Law 
and Technology Review (0001). http://www.law.duke.edu/journals/dltr/articles/ 2003dltr0001.html [25 May 2008].

The Economist. 2004. Make 'em pay: the fight against spam. 12 February: 74.

Turley SK. 1999. A case of response rate success. Journal of the Market Research Society 41(3): 301-310.

Van Zandt T. 2004. Information overload in a network of targeted communication. RAND Journal of Economics 35(3): 542-560.

\section{Appendix}

Table 2 summarizes parameter and stock values at steady state.

Table 2. Steady-state values and units

\begin{tabular}{|c|c|c|c|}
\hline & & Value & Units \\
\hline \multicolumn{4}{|c|}{ Recipients sector } \\
\hline$R$ & Number of recipients & 100 & Recipient \\
\hline & Average time recipient spends on messages & 20 & Minute/(day * recipient) \\
\hline & Normal response delay & 4 & Days \\
\hline & Average time to process a message & 1 & Minute/message \\
\hline $\bar{d}$ & Average sensitivity to overload & 3 & Dimensionless \\
\hline & Message backlog & 8830 & Messages \\
\hline & Valuable B messages in backlog & 492 & Messages \\
\hline$B$ & B messages in backlog & 6402 & Messages \\
\hline \multicolumn{4}{|c|}{ Sponsors sector } \\
\hline & Budget duration & 10 & Days \\
\hline & Fraction & 0.1 & Dimensionless \\
\hline$\omega$ & Value of a bond & 0 & \$/message \\
\hline & Marginal revenue & 2000 & \$/message \\
\hline & Communication funding & 202796 & $\$$ \\
\hline \multicolumn{4}{|c|}{ Broadcasters A sector } \\
\hline & Average flow of A messages & 10 & Messages/day/recipient \\
\hline \multicolumn{4}{|c|}{ Broadcasters B sector } \\
\hline & Base price & 1 & \$/message \\
\hline & Share of valuable B messages & 0.01 & Dimensionless \\
\hline & Surcharge & 0 & \$/message \\
\hline \multicolumn{4}{|c|}{ IT company sector } \\
\hline & Improvement delay & 10 & Days \\
\hline & Original $\eta_{1}$ & 0.01 & Dimensionless \\
\hline & Original $\eta_{2}$ & 0.12 & Dimensionless \\
\hline & Desirable aggregate misspecification rate & 0.1 & Dimensionless \\
\hline & Original aggregate misspecification rate & 0.1 & Dimensionless \\
\hline
\end{tabular}

